\title{
Smart Scheduling Strategy for Islanded Microgrid Based on Reinforcement Learning Algorithm
}

\author{
Lingxiao Gan ${ }^{\mathrm{a} *}$, Tao $\mathrm{Yu}^{\mathrm{a}}$, Jing $\mathrm{Li}^{\mathrm{a}}$, Jie Tang ${ }^{\mathrm{b}}$ \\ ${ }^{a}$ Electric Power College, South China University of Technology, Guangzhou 510640, China \\ ${ }^{b}$ Shaoguan Power Supply Bureau of Guangdong Power Grid Corporation, Shaoguan 512026, China
}

\begin{abstract}
This paper investigates a hierarchical Automatic Generation Control (AGC) strategy for an islanded microgrid, including wind power, solar photovoltaic, micro turbines, small hydropower and energy storage devices. The upper AGC is for central scheduling. The bottom AGC is to optimize the allocation factors, expecting to meet the requirement of energy-saving generation dispatching (ESGD). Three different bottom controllers are presented. Two of them are designed based on reinforcement learning (RL) algorithm. In order to evaluate their control performance, another proportion-based (PROP) controller which has been put into practical application is also presented. Detailed dynamic models of distributed generations and loads are built to simulate the microgrid. System responses to wind turbine tripping and to large load disturbances are tested. The results indicate that the proposed strategy based on RL algorithm can not only achieve reliability and stability of microgrid in islanded mode, but also reduce fossil energy consumption. This approach is a possible candidate for future microgrid control approaches.
\end{abstract}

Keywords: Distributed generation; islanded microgrid; hierarchical AGC; reinforcement learning algorithm

\section{Introduction}

Nowadays, energy-saving generation dispatching (ESGD) has been intended to be implemented in China, expecting to establish a long-term mechanism to promote clean energy development by improving the existing mode of dispatching [1]. The principle is that renewable and clean power generation resources can get a priority access, and dispatch the fossil energy resources from low to high in turn according to the level of energy consumption and pollutant emissions.

One of important features of smart grid is that the grid can accommodate to a wide variety of distributed low-carbon generations and storage options, achieving the coordinated control and optimal operation while minimizing operations and maintenance expenses [2], which is also central to the concept of microgrid. Traditional methods to design Automatic Generation Control (AGC) system is through system identification approach or engineering experience, whose application effect is limited because of uncertainties and time-varying operating conditions in modern power grid.

In this paper, on the foundation of dynamic models of distributed power sources, a hierarchical AGC strategy is proposed for an islanded microgrid. The upper AGC is for central scheduling. The bottom AGC is to optimize the allocation factors, expecting to meet the requirement of ESGD. Three different bottom controllers are presented. Two of them are designed based on reinforcement learning algorithm, and the third one is a proportion (PROP) controller. The MATLAB/Simulink simulation results show that the proposed control system based on reinforcement learning owns satisfied dynamic performance, makes the islanded microgrid operate safely and stably, and especially achieves the goal of ESGD.

\footnotetext{
* Manuscript received July 23, 2012; revised August 25, 2012.

Corresponding author. Tel.: +86-15013034721; E-mail address: ingxiaogan08@163.com.
} 


\section{Reinforcement Learning}

A reinforcement learning problem is an optimal control problem where the controller is given a scalar reinforcement signal indicating how well it is performing. The reinforcement signal is a function of the state of the system being controlled and the control signals chosen by the controller. The goal is to maximize the expected total discounted reinforcement [3]. One-step Q-learning is a simple incremental algorithm developed from the theory of dynamic programming. The multi-step $\mathrm{Q}(\lambda)$-learning, which is a new direct algorithm that extends the one-step Q-learning algorithm, propagates information rapidly to where it is important by "eligibility" trace, which may faster convergence of the algorithm [4].

\section{Distributed Generations Modeling}

\subsection{Small hydro generation model}

Small hydro power is clean, distributed and renewable energy. Although their available capacity is limited, small hydro generating units can be considered as base load units in a microgrid whose capacity is relatively small. The model of small hydro generation developed in [5] is used in this paper.

\subsection{Photovoltaic generation model}

PV systems have two big problems that the efficiency of electric-power generation is very low, especially under low-irradiation states, and the amount of the electric power generated by solar arrays is always changing with weather conditions. A detailed model of PV generation was described in [6]. Power output of photovoltaic generation in a single day is shown in Fig. 1.

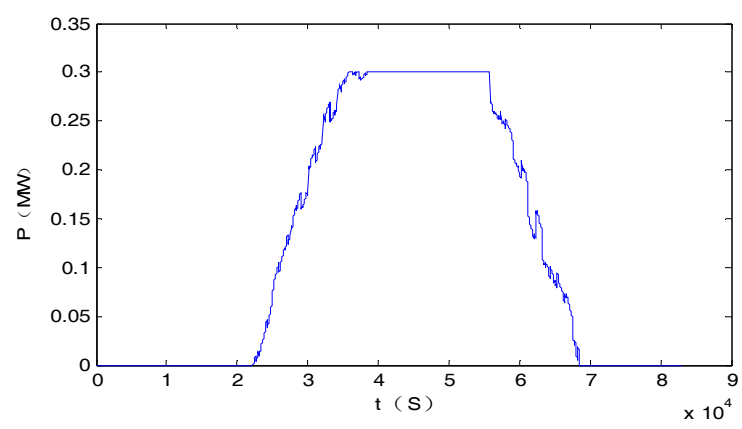

Fig.1. Daily power output of photovoltaic generations.

\subsection{Power output of wind generation considering tripping}

Recent years, because of lack of actual operating experience and wind farm design defects, wind turbine tripping accidents occur frequently in China. Taking that into consideration, simulation of power output of wind generation is shown in Fig.2. Wind turbines are tripped due to a system fault at $t=52,000 \mathrm{~s}$ that means power output of wind generation is reduced to zero, which may lead to mismatch between load and generation in the microgrid.

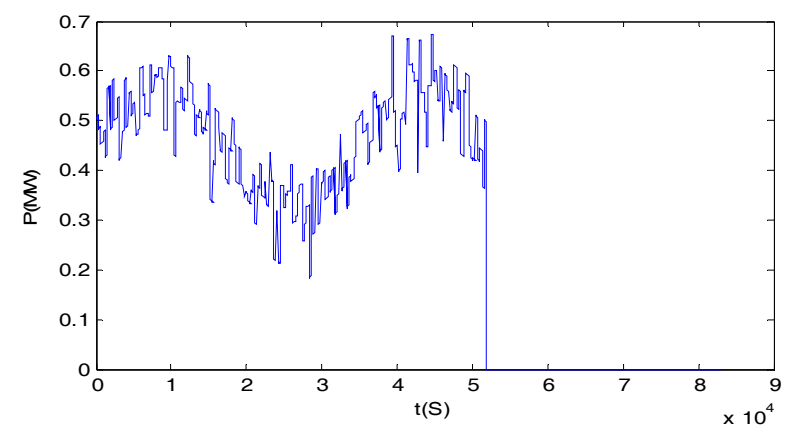

Fig. 2. Power output of wind generation considering tripping. 


\subsection{MT generation model}

Micro turbine (MT) generation is one of the most attractive distributed generation units. This is because micro turbines operate with less vibration and low noise, exhibit very fast response to load variation, require low maintenance, and run on a variety of fuels [7]. The model of MT is taken from [8].

\subsection{Energy storage devices model}

Energy storage devices are one of the main critical components to rely on for successful operation of a microgrid. Flywheels with enough power capacity are used in this paper. The detailed representation of a simulation model of flywheels was presented in [9].

\section{Simulation Cases and Analysis}

\subsection{Dispatch scheme}

Photovoltaic and wind power are important renewable sources whose major issue is the availability of their power is driven by weather, not the loads of the system, considered as non-dispatchable units. Power output intermittence of them and load fluctuation lead to non-planed instantaneous power in microgrid. Traditional dispatch scheme focuses on the stability and load tracking of the microgrid. However, taking ESGD into account, dispatch scheme will be different. Units of various kinds identify the energy-saving sorted order according to the following sequence: 1) wind and PV generation units; 2) small hydro generation units; 3) natural gas generation units (WT). A corresponding hierarchical AGC strategy is shown in Fig. 3. The upper AGC is for central scheduling. The bottom AGC for which further study will be made is to optimize the allocation factors.

\subsection{Bottom Q-controller design}

\section{A. State and action space discretization}

The degree of state and action space discretization plays an important role to the AGC performance of Q-controller. The state variable $u_{1}$ of Q-controller which is also the output signal of the upper AGC is discretized to the following 6 levels: $(-\infty,-2],(-2,-1.9],(-1.9,0],(0,1.9],(1.9,2](2,+\infty)$. The control actions, namely the output of bottom AGC, are the dynamic allocation factors. The action space is discretized into 25 values equal to $\{0.04,0.08,0.12, \cdots, 0.92,0.96,1\}$.

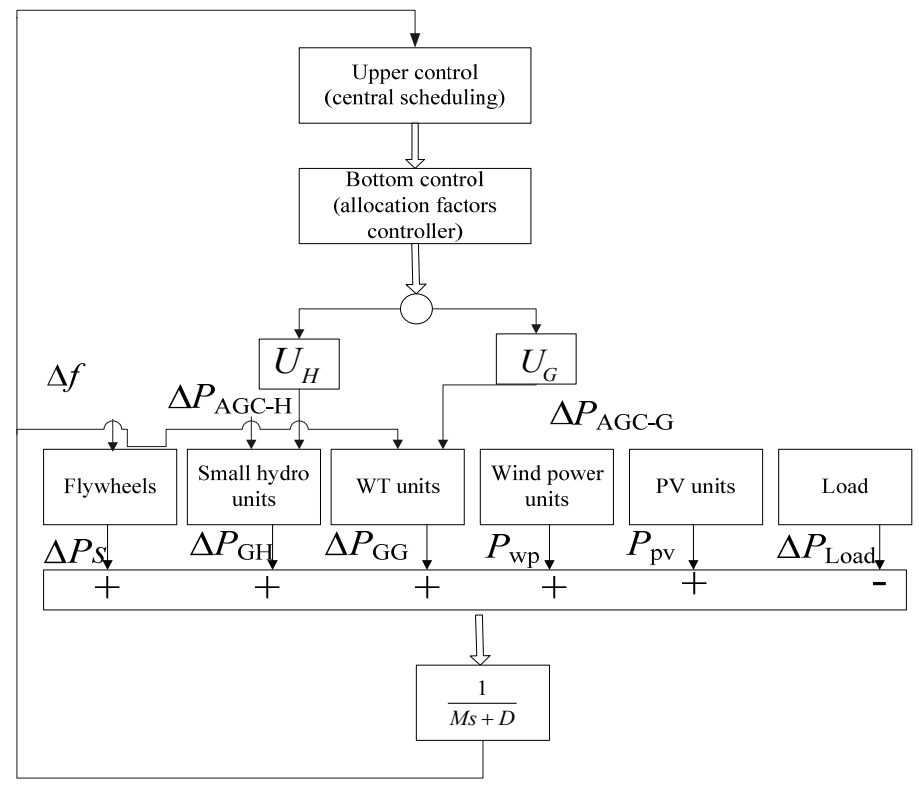

Fig. 3. Hierarchical AGC strategy for microgrid. 


\section{B. Reward function}

The control objective of the AGC scheme can be defined by the immediate reward function. ESGD is a multi-objective dynamic optimal problem. The primary objective is to regulate frequency to the specified nominal value by adjusting the output of selected generators. A secondary objective is energy conservation and environmental protection through allocating generations. In other words, the dispatch scheme always tries to minimize power output deviation and then makes the renewable and clean power sources used as much as possible. Based on that, a reward function is designed as follows:

$$
r_{i}(t)=\left\{\begin{array}{lc}
-\alpha\left[P_{\text {order- } \Sigma}(t)-P_{\mathrm{G} 1}(t)-P_{\mathrm{G} 2}(t)\right]^{2}+\beta_{2} \theta_{1}(t)-\gamma_{3} \theta_{2}(t) & u_{1} \leq-2 \\
-\alpha\left[P_{\text {order- } \Sigma}(t)-P_{\mathrm{G} 1}(t)-P_{\mathrm{G} 2}(t)\right]^{2}+\gamma_{2} \theta_{2}(t) & -2<u_{1} \leq-1.9 \\
-\alpha\left[P_{\text {order } \Sigma}(t)-P_{\mathrm{G} 1}(t)-P_{\mathrm{G} 2}(t)\right]^{2}+\beta_{1} \theta_{1}(t)-\gamma_{1} \theta_{2}(t) & -1.9<u_{1} \leq 0 \\
-\alpha\left[P_{\text {order- } \Sigma}(t)-P_{\mathrm{G} 1}(t)-P_{\mathrm{G} 2}(t)\right]^{2}+\beta_{1} \theta_{1}(t)-\gamma_{1} \theta_{2}(t) & 0<u_{1} \leq 1.9 \\
-\alpha\left[P_{\text {order }-\Sigma}(t)-P_{\mathrm{G} 1}(t)-P_{\mathrm{G} 2}(t)\right]^{2}+\gamma_{2} \theta_{2}(t) & 1.9<u_{1} \leq 2 \\
-\alpha\left[P_{\text {order }-\Sigma}(t)-P_{\mathrm{G} 1}(t)-P_{\mathrm{G} 2}(t)\right]^{2}+\beta_{2} \theta_{1}(t)-\gamma_{3} \theta_{2}(t) & 2<u_{1}
\end{array}\right.
$$

where

$P_{\text {order- } \Sigma}(t):$ central dispatch order, MW; $P_{\mathrm{G} 1}(t):$ Power output of small hydro units, MW;

$P_{\mathrm{G} 2}(t)$ : Power output of MT units, MW; $\theta_{1}(t)$ : Ratio of $P_{\mathrm{G} 1}(t)$ to $P_{\text {order- } \Sigma}(t), \%$;

$\theta_{2}(t)$ : Ratio of $P_{\mathrm{G} 2}(t)$ to $P_{\text {order }-\Sigma}(t), \% ; \alpha, \beta_{i=1,2}, \gamma_{i=1,2,3}$ : weight coefficients.

The goal of RL is to maximize the expected total discounted reinforcement. On one hand, microgrid frequency stability must be guaranteed first and then meet the requirement of ESGD, i.e., the deviation between load and generation should be minimized. According to that, a negative coefficient $(-\alpha)$ is set before the power deviation item. On the other hand, in order to encourage small hydro units to be the base load units, positive coefficients $\left(+\beta_{i=1,2}\right)$ are set before $\theta_{1}(t)$. Since the WT have rapid adjustment ability, they are expected to participate in peak load regulation and positive incentive factors $\left(+\gamma_{i=2}\right)$ are set before $\theta_{2}(t)$.

\section{Simulation case}

Dynamic simulation of the microgrid is established using Matlab/Simulink and the controller is implemented as S-Function module. Power output of PV and wind power generations was previously described in Fig. 1 and Fig. 2, respectively. Load curve is periodic sine-wave load as shown in Fig.4.

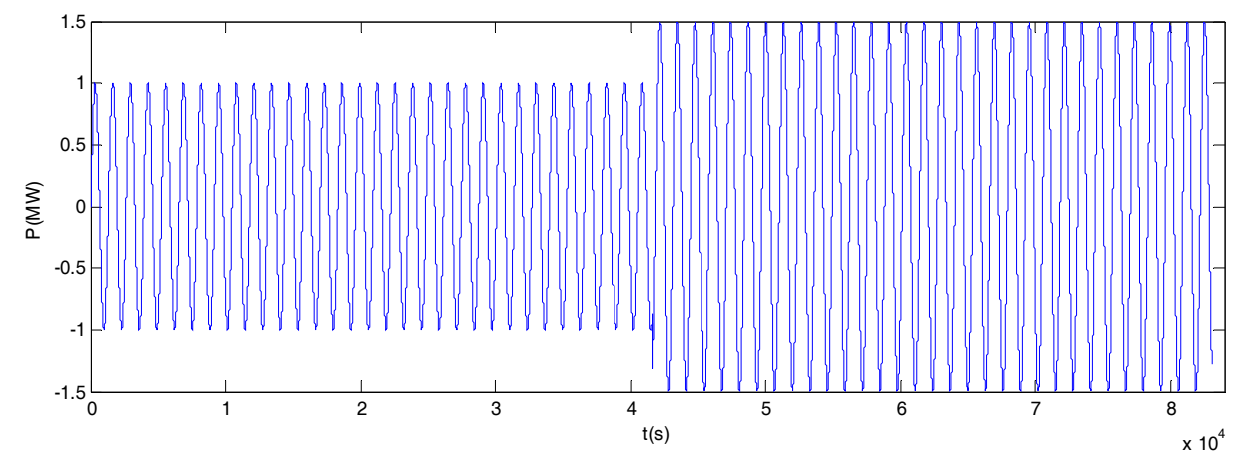

Fig.4 Load curve.

The simulation results are shown in Fig. 5. Normally, no experiences will be available in the initial stage for the RL driven controller to control the AGC system adequately. As a remedy, controllers should be scheduled to experience a series of trial-and-error procedures called "pre-learning stage", which is a stochastic action exploration process. After that, the total power output precisely tracks the changing load except for some special moments, though wind turbine tripping accident happened. The frequency deviation is smaller than $\pm 0.02 \mathrm{~Hz}$ except for the pre-learning stage, which is in the acceptable range. 


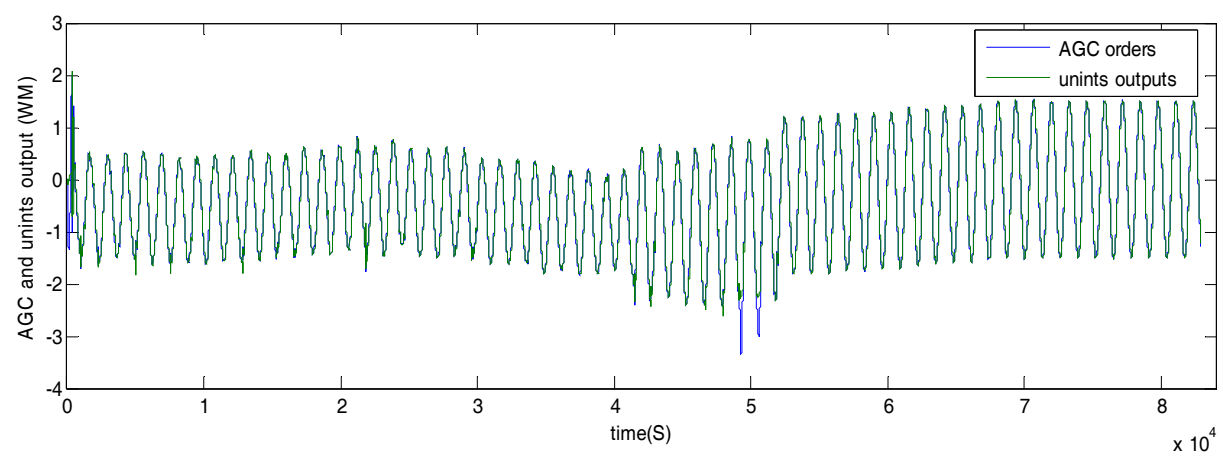

(a)

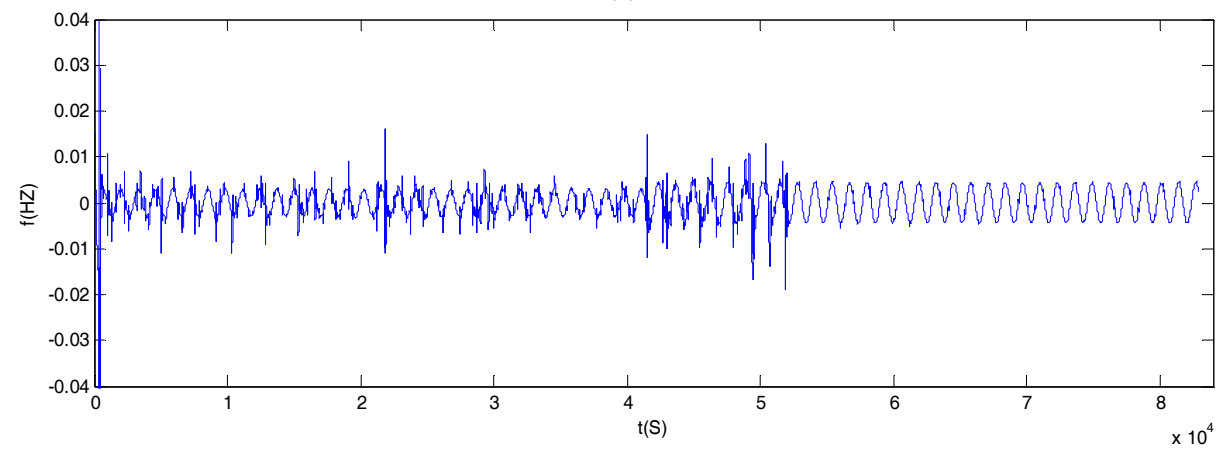

(b)

Fig.5. Power and frequency deviation with Q-controller.

\subsection{Bottom $Q(\lambda)$-controller design}

In order to compare the performance of Q-controller and $\mathrm{Q}(\lambda)$-controller, the state and action space discretization and reward function are the same. As is depicted in Fig. 6, the pre-learning stage of $Q(\lambda)$ controller is about 400 seconds, shorter than that of Q-controller. Similarly the power output follows precisely the changing load and the frequency deviation of microgrid is within $\pm 0.02 \mathrm{~Hz}$.

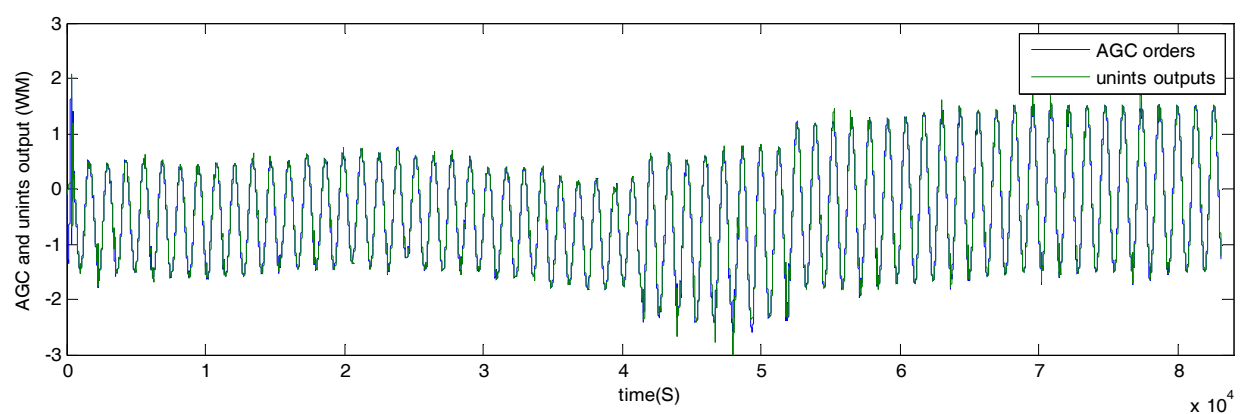

(a)

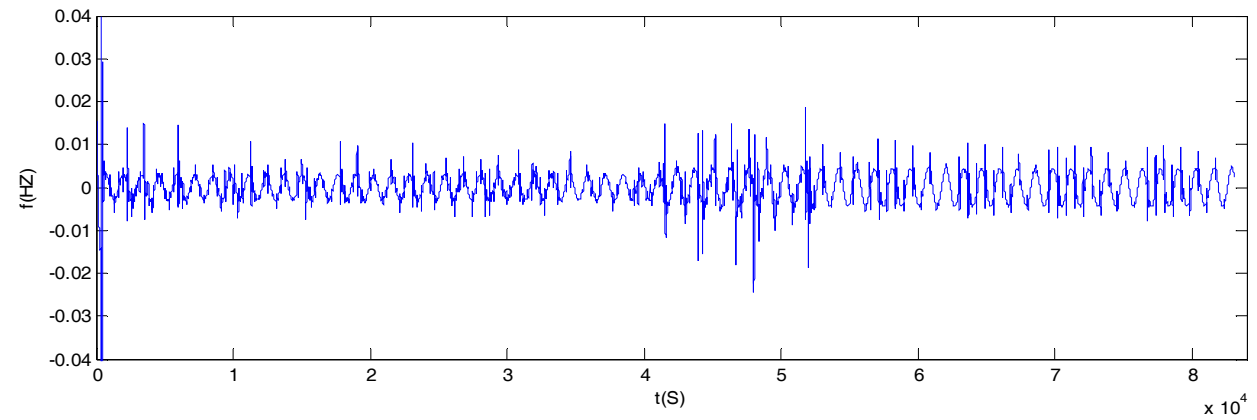

(b)

Fig. 6. Power and frequency deviation with $\mathrm{Q}(\lambda)$-controller. 


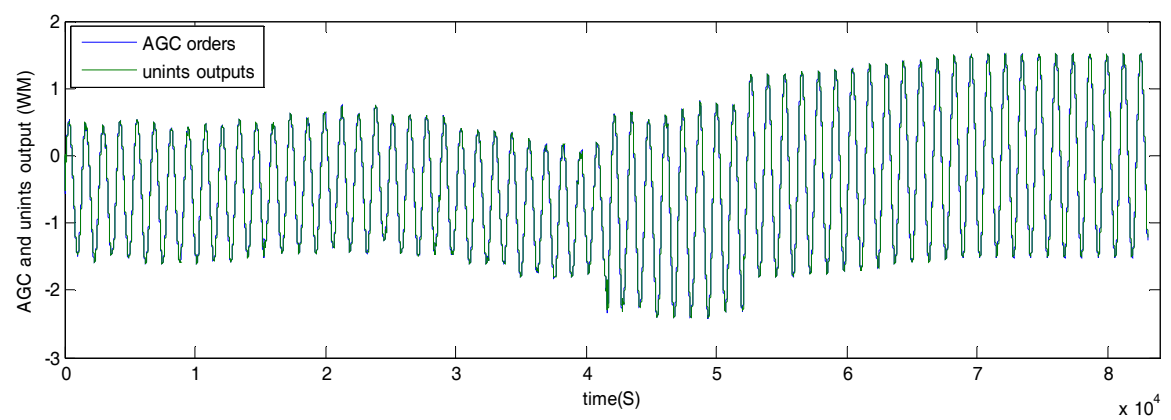

(a)

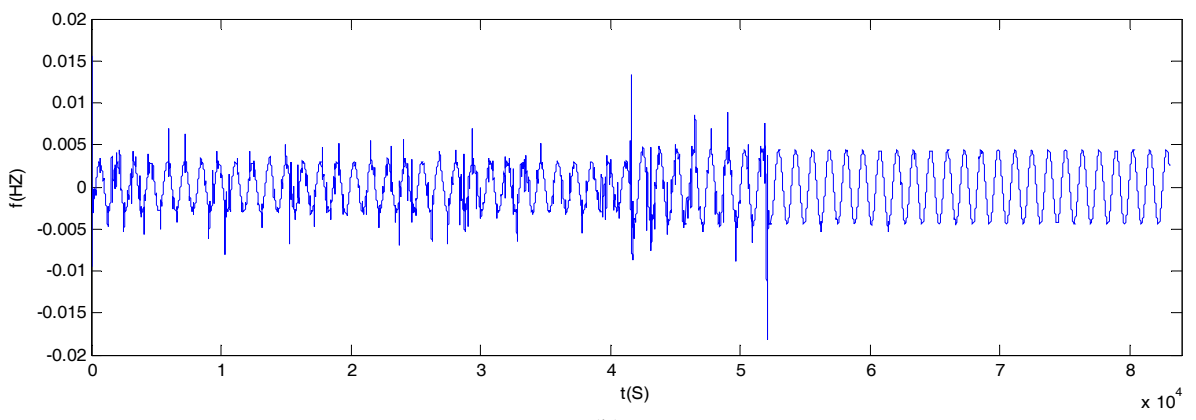

(b)

Fig. 7. Power and frequency deviation with PROP controller.

\subsection{Bottom PROP controller design}

PROP algorithm based on which allocation factors are fixed and proportional to adjustable capacity of each AGC unit, is used by NERI in their actual AGC system. Allocation factors of small hydro units and MT in this paper are $0.66(\mathrm{UH})$ and 0.34 (UG), respectively. The simulation results are shown in Fig. 7.

\subsection{Performance comparison of three controllers}

The steady-state frequency deviations with three different controllers are less than $\pm 0.02 \mathrm{~Hz}$. They all own good load following performance, but the convergence time of $\mathrm{Q}(\lambda)$-learning algorithm is shorter than Q-learning algorithm. In terms of ESGD, an evaluation criterion is assumed that under the same conditions the more hydro power and less WT generation are scheduled, the better the energy conservation will be. Total power output of WT is shown in Table 1. Total power output with Qcontroller and Q $(\lambda)$ - controller are more than $40 \%$ reduction than with PROP controller.

Table 1. WT's power output

\begin{tabular}{ccc}
\hline Controller & Power output $(\mathrm{kWh})$ & Reduction $(\%)$ \\
\hline PROP & 6807.60 & 0 \\
Q $(\lambda)$ & 4004.70 & 41.17 \\
Q & 3921.30 & 42.40 \\
\hline
\end{tabular}

Considering the frequency stability of the microgrid, load following and energy conservation, $Q(\lambda)$ controller with online learning and adaptive characteristics, is more effective and suitable to deal with strong randomness and ESGD issues of a microgrid.

\section{Conclusions}

Taking ESGD into account, a hierarchical AGC strategy based on reinforcement learning algorithm is proposed, which can achieve multi-objective dynamic optimal allocation of a microgrid in islanded mode.

RL algorithm is a typical stochastic optimal algorithm, applicable to AGC of power system which is a stochastic optimal decision-making problem, especially when systems contain distributed stochastic 
power sources, for example PV and wind power. The controllers based on RL have excellent online learning abilities and can adapt well to the random changes in the operating environment, which improves the adaptability and robustness of the system. Moreover the proposed control system meets the requirement of ESGD. It is a possible candidate for future microgrid control approaches.

\section{Acknowledgements}

This work was jointly supported by the National Natural Science Foundation of China (No. 50807016, 51177051), Science and technology projects of China Southern Power Grid, the Fundamental Research Funds for the Central Universities (2012ZZ0020),open fund of state key laboratory sponsored by Tsinghua University (SKLD10KM01).

\section{References}

[1] Xie GH, Zhang LZ, He XY, Research on the model and method for energy-saving generation dispatching, In: Proc. of International Conference on Sustainable Power Generation and Supply, 2009:1-7.

[2] Vaziri M, Vadhva S, Oneal T, Johnson M, Smart grid, distributed generation, and standards, In: Proc. of Power and Energy Society General Meeting, 2011:1-8.

[3] Baird LC III, Reinforcement learning in continuous time: advantage updating. In: Proc. of IEEE World Congress on Computational Intelligence Neural Networks, 1994:2448-2453.

[4] Jing P, Ranold JW, Incremental multi-step Q-learning, Machine Leaning, 1996; 22(1-3):283-290, 1996.

[5] Kundur P. Power System Stability and Control, New York: McGraw-Hill; 1994.

[6] Saito N, Niimura T, Koyanagi K, et al. Trade-off analysis of autonomous microgrid sizing with PV, diesel, and battery storage, In: Proc. of IEEE Power \& Energy Society General Meeting, 2009:1-6.

[7] Laili MS, Zakaria ZN, Halim NH, Ibrahim P. Modelling and simulation of microturbine for a distribution system network with hybrid filter. In: Proc. of IEEE International Power Engineering and Optimization Conference, 2012:204-208.

[8] Yu T, Zhou B, Chan KW, et al. Stochastic optimal relaxed automatic generation control in Non-Markov environment based on multi-step Q $(\lambda)$ learning. IEEE Trans. on Power Systems, 2011; 26(3):1272-1282.

[9] Awad B, Ekanayake JB, Jenkins N. Intelligent load control for frequency regulation in microgrids. Journal of Intelligent Automation \& Soft Computation, 2008; 16(2):303-318. 